\title{
Center for Extended Magnetohydrodynamic Modeling Cooperative Agreement
}

\author{
Final Report \\ February 15, 2002 - February 14, 2008 \\ University of Wisconsin-Madison, Madison, WI 53706 \\ in cooperation with
}

Princeton Plasma Physics Laboratory, Massachusetts Institute of Technology, New York University, Utah State University, Science Applications International

Corporation, Tech-X Corporation, and the University of Colorado

\author{
Institutional Principal Investigator: Carl R. Sovinec \\ Lead Principal Investigator: Stephen C. Jardin, PPPL
}

May, 2008

\author{
THE U.S. DEPARTMENT OF ENERGY \\ AWARD NO. DE-FC02-02ER54668
}

\begin{abstract}
NOTICE
This report was prepared as an account of work sponsored by the United States Government. Neither the United States Government, nor any agency thereof, nor any of their employees, makes any warranty, express or implied, or assumes any legal liability or responsibility for the accuracy, completeness, or usefulness of any information, apparatus, product or process disclosed, or represents that its use would not infringe privately-owned rights. Reference herein to any specific commercial product, process, or service by trade name, trademark, manufacturer, or otherwise does not necessarily constitute or imply its endorsement, recommendation, or favoring by the United States Government or any agency thereof. The views and opinions of authors expressed herein do not necessarily state or reflect those of the United States Government or any agency thereof.
\end{abstract}




\section{Executive Summary}

The Center for Extended Magnetohydrodynamic Modeling (CEMM) is developing computer simulation models for predicting the behavior of magnetically confined plasmas. Over the first phase of support from the Department of Energy's Scientific Discovery through Advanced Computing (SciDAC) initiative, the focus has been on macroscopic dynamics that alter the confinement properties of magnetic field configurations. The ultimate objective is to provide computational capabilities to predict plasma behavior-not unlike computational weather prediction - to optimize performance and to increase the reliability of magnetic confinement for fusion energy. Numerical modeling aids theoretical research by solving complicated mathematical models of plasma behavior including strong nonlinear effects and the influences of geometrical shaping of actual experiments. The numerical modeling itself remains an area of active research, due to challenges associated with simulating multiple temporal and spatial scales. The research summarized in this report spans computational and physical topics associated with state of the art simulation of magnetized plasmas.

The tasks performed for this grant are categorized according to whether they are primarily computational, algorithmic, or application-oriented in nature. All involve the development and use of the Non-Ideal Magnetohydrodynamics with Rotation, Open Discussion (NIMROD) code [1], which is described at http://nimrodteam.org. With respect to computation, we have tested and refined methods for solving the large algebraic systems of equations that result from our numerical approximations of the physical model. Collaboration with the Terascale Optimal PDE Solvers (TOPS) SciDAC center led us to the SuperLU_DIST software library for solving large sparse matrices using direct methods on parallel computers [2]. Switching to this solver library boosted NIMROD's performance by a factor of five in typical large nonlinear simulations, which has been publicized as a success story of SciDAC-fostered collaboration [3]. Furthermore, the SuperLU software does not assume any mathematical symmetry, and its generality provides an important capability for extending the physical model beyond magnetohydrodynamics (MHD).

With respect to algorithmic and model development, our most significant accomplishment is the development of a new method for solving plasma models that treat electrons as an independent plasma component. These 'two-fluid' models encompass MHD and add temporal and spatial scales that are beyond the response of the ion species. Implementation and testing of a previously published algorithm did not prove successful for NIMROD, and the new algorithm has since been devised, analyzed, and implemented. Two-fluid modeling, an important objective of the original NIMROD project, is now routine in 2D applications. Algorithmic components for 3D modeling are in place and tested; though, further computational work is still needed for efficiency. Other algorithmic work extends the ion-fluid stress tensor to include models for parallel and gyroviscous stresses. In addition, our hot-particle simulation capability received important refinements that permitted completion of a benchmark with the M3D code [4].

A highlight of our applications work is the edge-localized mode (ELM) modeling, which was part of the first-ever computational Performance Target for the DOE Office of Fusion Energy Science, see http://www.science.doe.gov/ofes/performancetargets.shtml. Our efforts allowed MHD simulations to progress late into the nonlinear stage, where energy is conducted to the wall location. They also produced a two-fluid ELM simulation starting from experimental information and demonstrating critical drift effects that are characteristic of two-fluid physics. Another important application is the internal kink mode in a tokamak. Here, the primary purpose of the study has been to benchmark the two main code development lines of CEMM, NIMROD and $\mathrm{M} 3 \mathrm{D}$, on a relevant nonlinear problem. Results from the two codes show repeating 
nonlinear relaxation events driven by the kink mode over quantitatively comparable timescales [5]. The work has launched a more comprehensive nonlinear benchmarking exercise, where realistic transport effects have an important role.

\section{Goals and Accomplishments}

In this section, we list the primary goals taken from the scope of work statements for the funding periods covered by this final report. Relevant accomplishments are described after each of the bulleted goals.

Improve and expand linear solvers:

- Complete linear solver implementation for non-Hermitian matrices.

We first performed tests with the AZTEC software library [http://www.cs.sandia.gov/CRF/aztec1.html] from Sandia National Laboratory. However, after testing by collaborators from the TOPS center showed favorable performance with parallel direct methods, we implemented a coupling to the parallel SuperLU_DIST [2] software library and found a large gain in computational performance in simulations with Hermitian matrices. The coupling also provided capability for solving non-Hermitian matrices.

- Investigate different iterative methods for solving 3D matrix-free nonsymmetric systems.

A block-Jacobi algorithm that used the parallel SuperLU software to invert large diagonal blocks was implemented and tested. Later, NIMROD Team member D. C. Barnes of the University of Colorado-Boulder implemented a GMRES algorithm that used the parallel SuperLU solver to precondition matrix-free Krylov iterations. We further refined the implementation to reduce temporary storage and to expand preconditioning options.

Develop two-fluid modeling capability:

- Begin algorithm development for simulating two-fluid sets of equations via semi-implicit methods.

We implemented a semi-implicit algorithm for advancing the magnetic field with the Hall effect. Tests produced favorable results for electron-MHD (where ions are fixed), but numerical instability resulted in Hall-MHD computations. We performed numerical analysis and proved that the algorithm is unstable. The analysis led to the development of the new implicit leapfrog algorithm.

- Complete development for accurate simulation of Hall and gyroviscous effects with timesteps that are suitable for long time-scale phenomena.

We have completed the implementation of the implicit leapfrog algorithm including linear and nonlinear parts of the Hall term, gyroviscous stress, and implicit advection. Linear contributions to magnetization heat fluxes for separate electron and ion temperatures have also been implemented. Nonlinear coefficients are centered in the advance of each physical field by temporal staggering of different advances, by averaging, or by Newton-like linearization from the beginning of the advance.

Improve computational performance for flow:

- Improve performance in conditions of strong flow and low dissipation by implementing discontinuous basis functions for the predictor-corrector advection algorithm.

Analysis indicates that the proposed algorithm is too dissipative for use in simulations of magnetically confined plasmas. Instead, a nonlinear artificial diffusion term that acts in the 
direction of flow has been implemented for the implicit flow algorithm (described next). It corrects dispersive errors like upwinding, but it does not require distortion of test functions.

- Begin testing non-ideal boundary and implicit equilibrium flow capabilities.

We implemented 3D temperature-dependent resistivity that reproduces the effect of an external vacuum region in locations that are thermally connected to a cold wall. Effects of a non-ideal wall located outside the vacuum region have not been fully implemented, however. We performed numerical analysis of time-centered implicit advection and found that it is compatible with semi-implicit MHD. It was successfully tested on linear computations with time-steps exceeding the explicit numerical stability limit.

- Investigate use of a matrix-free nonsymmetric solver capability for fully implicit flow computations.

We performed numerical analysis and found that the implicit leapfrog algorithm requires implicit advection to avoid severe time-step constraints. The advance of each physical field was modified accordingly without losing the option to perform nonlinear MHD with predictor-corrector advection. The matrix-free GMRES algorithm described in the linearsolver section has proven to be effective in nonlinear MHD simulations with implicit advection.

Expand modeling capability for fluid stresses:

- Modify the ion viscous stress tensor to include a parallel component.

The fluid-based parallel viscous stress has been implemented, including contributions from equilibrium and perturbed flows. The implementation is implicit for numerical stability, and it relies on 3D matrix-free iterative solves.

- Implement parallel electron viscous stress effects modeled with differential operators.

We have implemented the linear part of parallel electron viscous stress in the advance of magnetic field. As a fourth-order differential operator in space, the implementation uses an auxiliary scalar field that is solved simultaneously with the change in magnetic field. The effect of this stress has been verified in whistler-wave tests, where analytics show that damping from the electron stress is greatest for propagation at intermediate angles with respect to the background magnetic field.

- Work with the Utah State group to facilitate the use of the differential electron stress as a semi-implicit operator for nonlocal stress models.

We have collaborated with E. D. Held and J.-Y. Ji of Utah State University, including hosting them as visiting scientists at the University of Wisconsin while on sabbatical.

Improve implementation of hot-particle effects:

- Upgrade the hot-particle simulation capability to allow its use with high-order finite elements.

The particle simulation capability was modified to use fields with high-order finite elements. Improvements to the associated kinetic stress computation and trajectory integrator contributed to a successful verification on results from the M3D code for an internal kink/fishbone mode. Improvements in domain decomposition for parallel computing made the algorithm more efficient.

Improve meshing: 
- Explore mesh distortion as a means to improve the resolution of varying and evolving spatial scales.

The mapping and equilibrium fields were modified to Lagrange polynomial bases instead of the cubic splines used in the original NIMROD implementation. This increased flexibility and reliability when using nonuniform meshing. Dynamically adaptive mesh distortion has not yet been explored. The need for a completely new algorithm for two-fluid effects had not been anticipated and was given higher priority.

Exercise recent developments in research computations:

- Initiate a simulation study of neoclassical tearing modes using computational geometries and parameters that are realistic for high-performance discharges in tokamaks.

A nonlinear computation of a classical tearing mode demonstrated the numerical ability to run relatively realistic simulations (large separation of resistive and Alfvénic time scales, $\mathrm{S}=10^{6}$, and low viscosity, $\mathrm{Pm}=0.1$ ) for tearing behavior over long transport-relevant time scales. Linear two-fluid tearing calculations in slab geometry have been used for verification with analytical theory in the limit of large guide-field. Nonlinear neoclassical tearing requires the implementation of nonlinear electron viscosity, which has not been completed.

- Applications will test extended modeling capabilities for tokamak physics.

The edge-localized mode (ELM) computations for the OFES performance targets of FY0506 demonstrated two-fluid drift stabilization of short wavelength modes, an essential aspect of laboratory results. In addition, they demonstrated the suitability of the implicit leapfrog algorithm for large nonlinear problems. However, decreasing effectiveness of the algebraic solver as the perturbation amplitude increased in the nonlinear two-fluid simulation brought attention to the need for extending preconditioning to address coupling among Fourier components.

- Apply newly developed two-fluid capabilities to the $1 / 1$ tokamak mode.

The 1/1 internal kink mode in conditions relevant to the CDX-U tokamak has been used as a nonlinear benchmark application with the M3D code. We have also used the two-fluid algorithm on a cylindrical tokamak kink mode. Here, the limitations of the algebraic solver that hampered the ELM application were also problematic.

\section{Summary}

The objective of the NIMROD code development effort is to produce computational simulation software for modeling macroscopic dynamics in magnetized plasmas. At the beginning of this SciDAC support, the MHD modeling algorithm was mature and in use for several applications despite performance limitations associated with available numerical linear algebra software [6]. As intended, the CEMM collaboration helped advance NIMROD and other codes into extended-MHD modeling.

\subsection{Two-fluid algorithm}

The most significant product of this grant is the development and implementation of a new algorithm for solving the two-fluid model for macroscopic dynamics in magnetically confined plasmas. Our initial efforts were to implement the semi-implicit Hall advance that is described in Ref. 7. The implementation used an auxiliary vector in the system of equations, so that the low-order continuity of our finite element basis functions would not be problematic. The implementation worked well for electron-MHD, where the ions are fixed, and we were able to reproduce whistler waves. However, tests with dynamic ions resulted in numerical instability. 
Mathematical analysis confirmed that the algorithm is numerically unstable. We then devised a new algorithm which is stable for arbitrarily large time-step for the full two-fluid model [8]. Figure 1 shows a comparison of results of numerical analysis for the semi-implicit Hall and the new 'implicit leapfrog' methods. The new algorithm retains the temporal stagger of the flow velocity from other fields, as in our semi-implicit MHD algorithm, but each field is advanced implicitly, including an implicit treatment of advection. Also unlike semi-implicit methods, the implicit operators are not symmetric, and algebraic solvers for non-Hermitian matrices (see Section 3.2) are required. Relative to the time-centered implicit method, the staggering of the implicit leapfrog leads to smaller algebraic systems, which can be more efficient computationally.

All essential elements of the two-fluid model in NIMROD have been completed for the implicit leapfrog advance. Linear and nonlinear contributions to the Hall electric field, Braginskii gyroviscosity, and implicit advection are fully implemented and tested. Linear contributions for magnetization heat flows arising from separate electron and ion temperatures have also been implemented. In addition, linear and nonlinear contributions to parallel viscous ion stress and the linear part of parallel viscous electron stress have been implemented using an implicit formulation that is consistent with the implicit leapfrog method. (The parallel stresses are not magnetization effects, but they are also important extensions to the MHD model.)
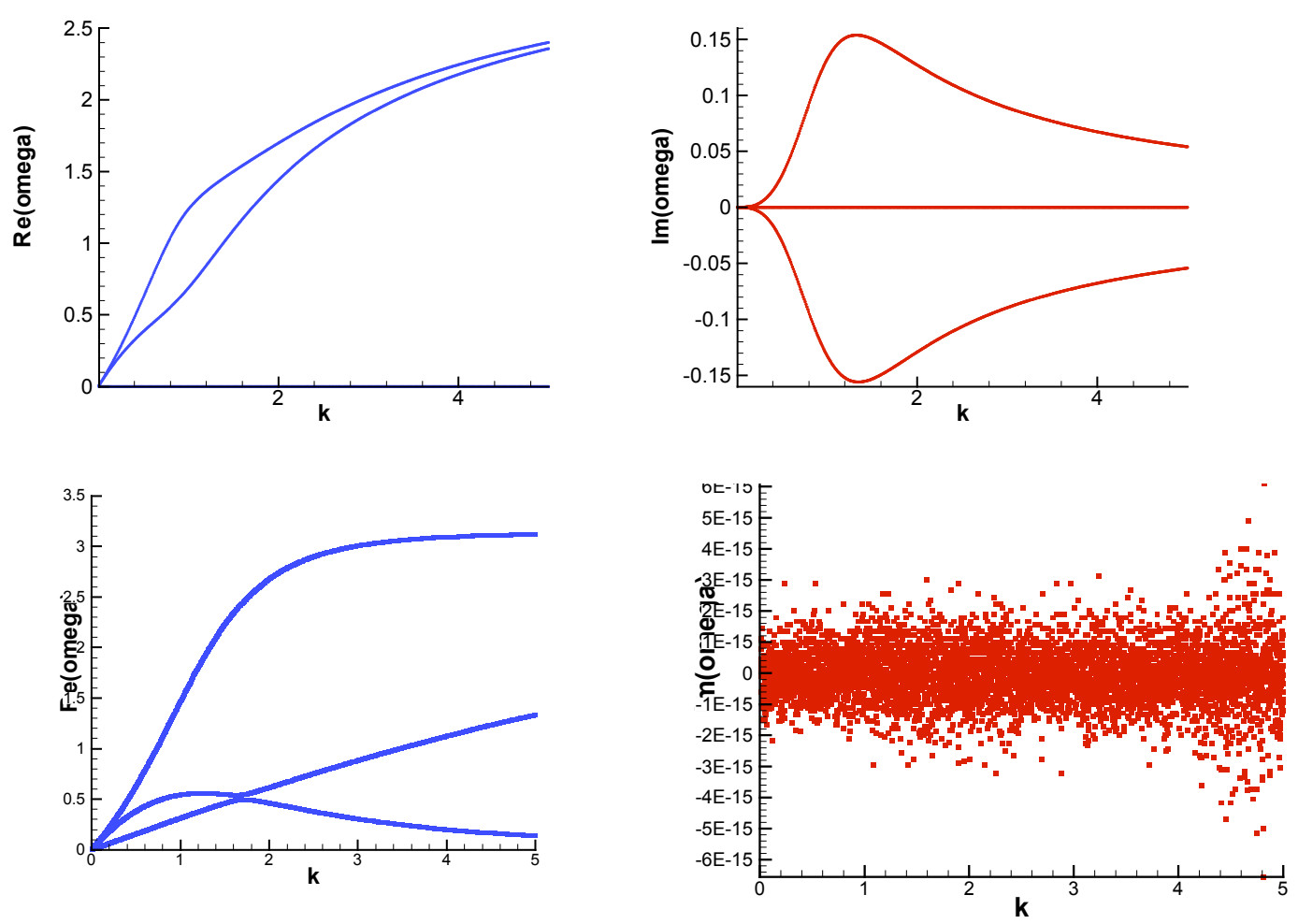

Figure 1. Real (left) and imaginary (right) parts of numerical frequencies for HMHD waves from analysis for the semi-implicit Hall advance at parallel propagation (top row) and for the implicit leapfrog advance for nearly parallel propagation (bottom row). Nontrivial positive imaginary values indicate numerical instability.

[http://www.cptc.wisc.edu/sovinec_research/meetings/sovinec_aps05poster.pdf] 


\section{$\underline{3.2 \text { Algebraic solvers }}$}

The disparity of temporal scales in macroscopic dynamics of magnetically confined plasmas requires implicit numerical methods to evolve solutions over dynamical scales without severe time-step restrictions associated with numerical stability. The implicit methods lead to large algebraic systems that need to be solved in parallel at each step. Preconditioners developed within the NIMROD Team had provided acceptable performance for some MHD computations. However, a very significant improvement resulted from SciDAC-fostered collaboration with the TOPS applied math and computer science research group [http://www.scidac.gov/ ASCR/ASCR_TOPS.html]. Their tests of matrices generated in NIMROD simulations demonstrated the effectiveness of the recently developed SuperLU_DIST software [2] for solving our sparse matrices in parallel. With help from Dr. Xiaoye Li, the author of SuperLU_DIST, we implemented a coupling for production computations. In nonlinear simulations, the direct solves are used on large independent blocks of the full matrix as a preconditioner for Krylov-space algorithms, and overall code performance improved by factors of 4-5. (See Figure 2.) In linear computations, where the direct algorithm inverts entire matrices and factoring is performed only once, CPU times dropped by two orders of magnitude. In addition, the SuperLU algorithm does not assume symmetry, allowing the first use of nonHermitian matrices in NIMROD.

Software for solving non-Hermitian systems in nonlinear two-fluid problems was developed by NIMROD Team member Dr. Daniel Barnes of the University of Colorado-Boulder. He added to NIMROD's conjugate gradient algorithm to allow use of the generalized minimal residual method (GMRES) with SuperLU for preconditioning. The Wisconsin group later added polynomial preconditioning and the use of a Hessenberg matrix to reduce storage.

\section{SuperLU DIST}

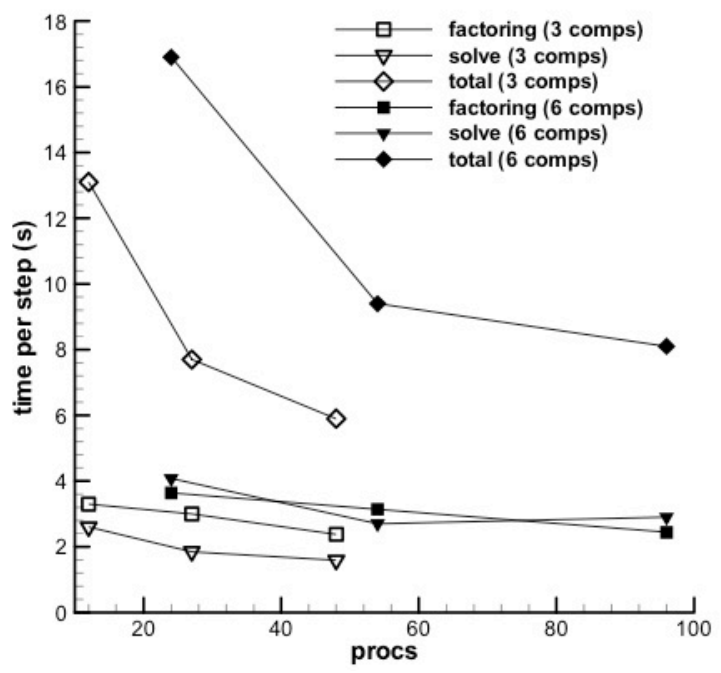

$\mathrm{cg}$ with line-Jacobi preconditioning

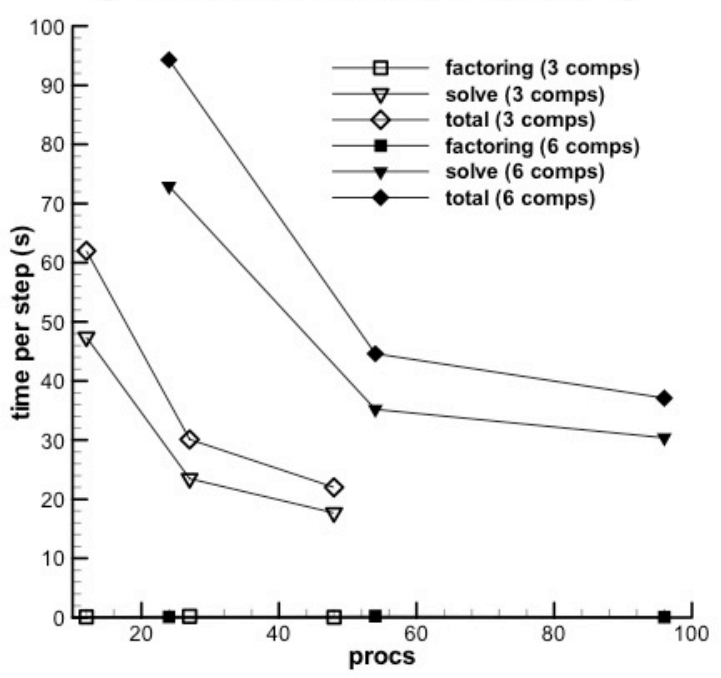

Figure 2. Comparison of parallel scaling performance with the SuperLU_DIST library shown on the left and with the native preconditioner for NIMROD on the right. The nonlinear MHD computation has a $32 \times 32$ mesh of biquartic finite elements, and the number of toroidal Fourier harmonics are indicated for different cases in the plots. On average, the matrices and factors are recreated every eight time-steps, and the timings are taken from an IBM SP3. 


\subsection{Simulation-particle development}

The author of the hot-ion particle kinetics module in NIMROD, Dr. Charlson Kim, spent part of the grant period as a postdoctoral associate in the Wisconsin group. During that time, the module was upgraded in several important ways [9]. He made the kinetic stress computation consistent with the finite element representation used for electromagnetic fields and later modified it to use integration by parts to reduce noise from spatial derivatives of moments of the simulation-particle distribution. He modified the particle advance to use a more accurate algorithm. He also made the domain decomposition for parallel computation more flexible, so that load balancing does not rely on having the same number of particles in all elements, which may vary in size. These developments contributed to bringing NIMROD's results into agreement with results from M3D on a tokamak 1/1 benchmark computation (Figure 3).

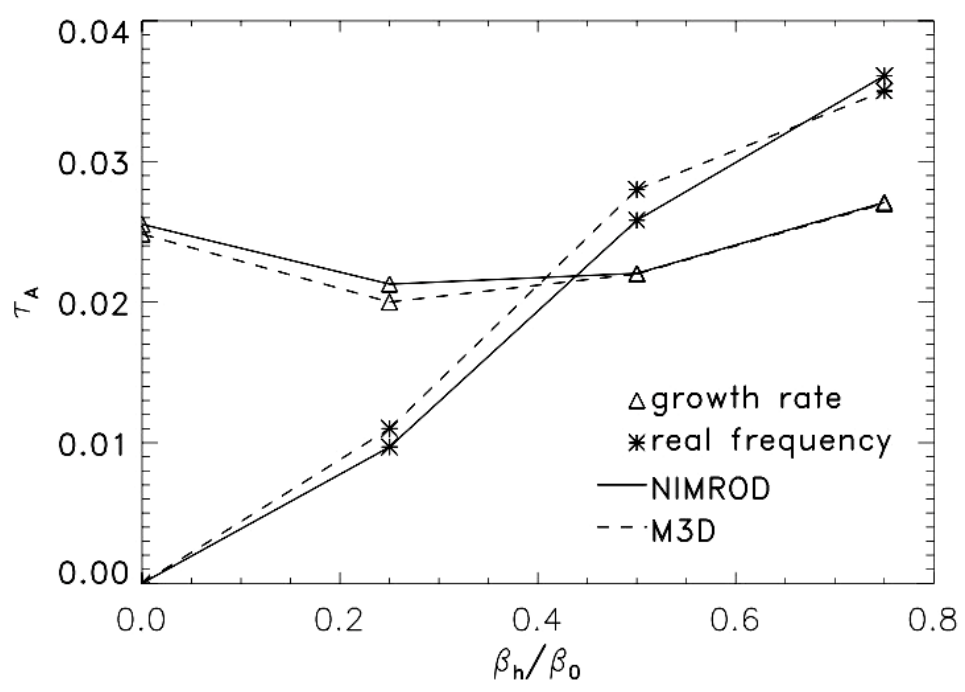

Figure 3. Comparison of growth rate and rotation frequency for a benchmark series of computations with the NIMROD and M3D codes using hot-ion kinetics. The horizontal axis displays the fraction of pressure from the hot-ion species.

[From https://nimrodteam.org/meetings/team_mtg_7_07_web/kim_team_mtg_7_07.pdf]

\subsection{Selected applications}

With the development and implementation of the implicit leapfrog algorithm and the progress on algebraic solvers, 2D two-fluid computations have become routine. Results on magnetic reconnection in the MRX experiment [10] and on natural rotation in field-reversed configurations [11] have been obtained using the implementation, and they have been published in the journal Physics of Plasmas. A study of drift stabilization for interchange modes, based on both analytics and numerical computation with NIMROD, considers how the effect is sensitive to equilibrium conditions [12]. This work has been submitted to Physical Review Letters. Computations illustrating drift effect in the nonlinear stage of interchange in slab configurations have also been performed (Figure 4). 


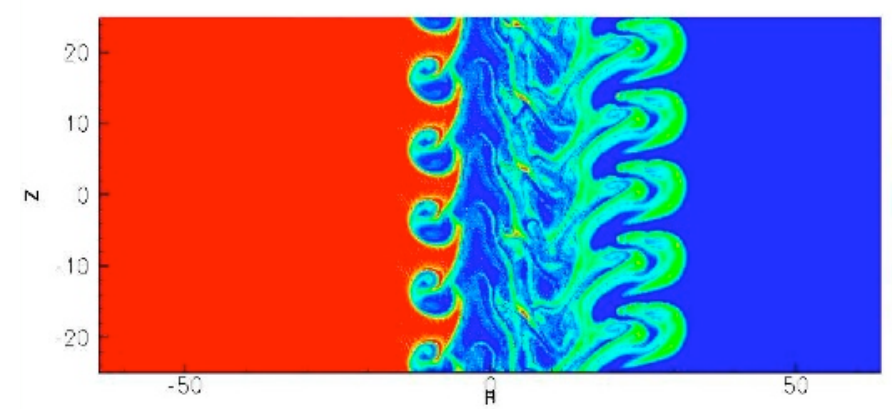

Figure 4. Contours of pressure from the nonlinear stage of interchange evolution in slab geometry, as computed by NIMROD with the implicit leapfrog model. [https://nimrodteam.org/meetings/team_mtg_7_07_web/zhu_blob_2fl_emb.pdf]

For magnetic confinement, the large guide-field limit of magnetic tearing is the most relevant condition. They are also the most computationally challenging for a full-dynamical model, because they induce the greatest separation of temporal scales. Graduate student Jacob King has benchmarked NIMROD's new algorithm with analytical theory for linear two-fluid tearing in this limit [13]. In large pressure and weakly unstable conditions that are relevant to many experiments, growth rates from theory and computation agree to within $0.5 \%$ when the resistive skin depth is $5 \%$ of the length-scale for shear in the slab-geometry equilibrium. The discrepancy increases with increased resistivity, because the resistive skin depth approaches the equilibrium length, violating the scale separation that is assumed in the analytical theory. When considering a range of pressures, simulations in the small-pressure limit reproduce the MHD growth rate and follow the two-fluid theory at increasing pressure, as shown in Figure 5. Nonlinear twodimensional two-fluid computations have also been successfully performed for this configuration.

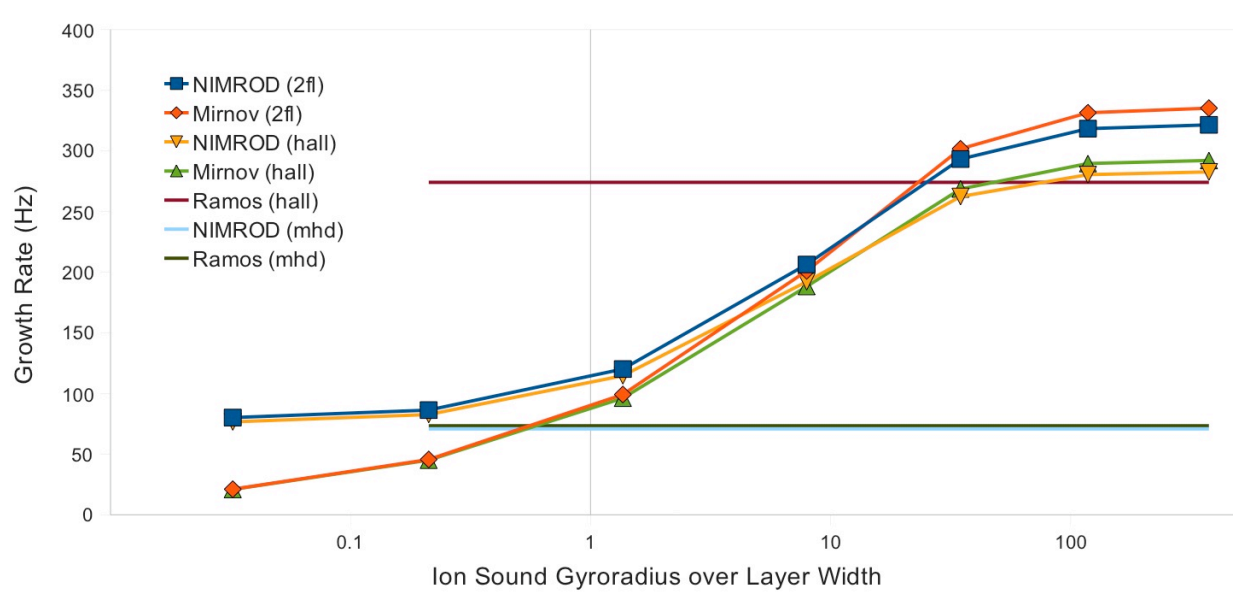

Figure 5. Linear verification results for two-fluid tearing in the large guide field limit. Computed NIMROD results on how the tearing growth rate changes with pressure are plotted together with analytical results by Mirnov that are valid away from small pressure limit (left side of figure) and with results computed by Ramos for the small and large pressure limits. The ' $2 \mathrm{fl}$ ' and 'Hall' indications refer to whether electron inertia is included ('2 $2 \mathrm{fl}$ '). 
Computations for the OFES FY05-06 Performance Target on simulating ELMs culminated in two-fluid modeling based on a profile that was fitted to measurements from the DIII-D tokamak at General Atomics [w3.pppl.gov/cemm/Milestones/FINAL_DRAFT_elm_milestone_06.pdf]. We performed both linear and nonlinear computations, and drift stabilization at high toroidal wavenumber, shown in Figure 6, is an important feature of the results. The spectrum of linearly unstable modes was used to initialize a nonlinear two-fluid computation that included the Hall effect and gyroviscosity. The numerical nonlinear artificial diffusivity described in Section 2 was used to avoid numerical dispersion from the implicit flow algorithm as the perturbations distorted the large density gradient in the edge region. Nonlinear coupling from the linearly unstable modes generated harmonic perturbation bands at low and high toroidal wavenumber (Figure 7), leading to the helically localized patterns shown in Figure 8. However, the lack of toroidal coupling in the preconditioning operation made the algebraic solver progressively less effective on the magnetic field advance with Hall term as the perturbation amplitude grew. Addressing this limitation is an important aspect of the follow-on phase of the research effort.

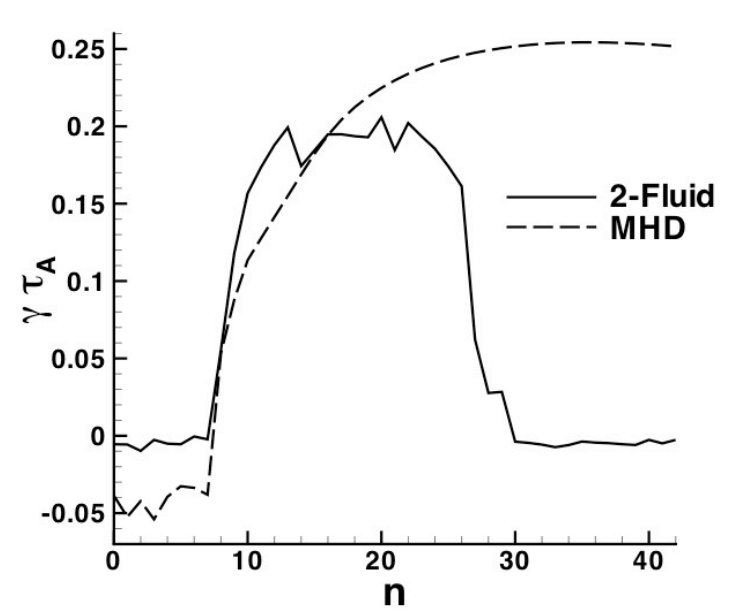

Figure 6. Linear growth-rate spectra of ELMs computed by NIMROD with the twofluid and MHD models for a realistic equilibrium. The horizontal axis indicates toroidal wavenumber.

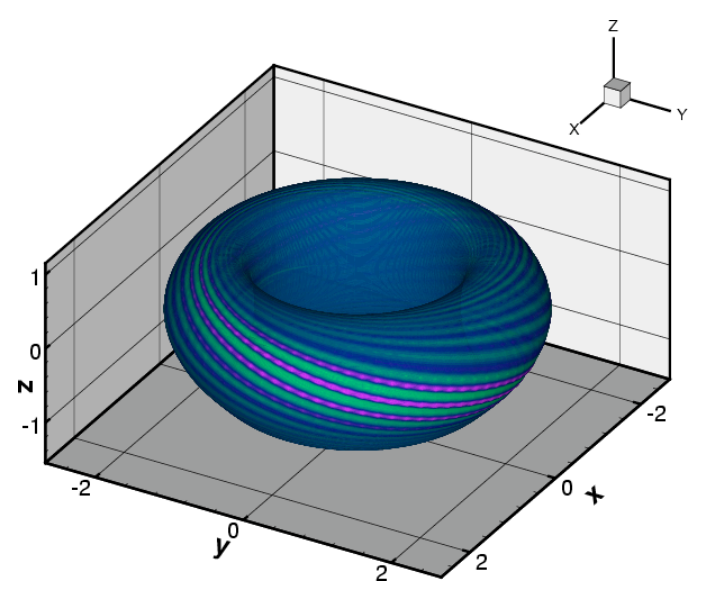

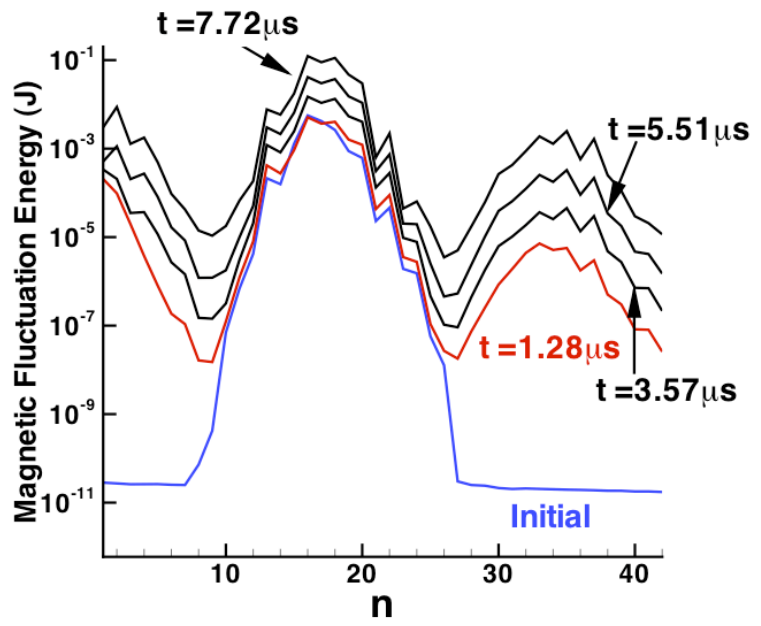

Figure 7. Evolution of the magnetic fluctuation spectrum from the nonlinear NIMROD ELM simulation with the twofluid model.
Figure 8. Temperature perturbations from the nonlinear two-fluid ELM simulation display helical localization at $25 \%$ amplitude with respect to the initial pedestal temperature. 
The Wisconsin group has also participated in the benchmarking of NIMROD and M3D for nonlinear resistive MHD evolution from the tokamak 1/1 internal kink. The problem was defined to be relevant to the CDX-U tokamak, where realistic parameters are tractable for both codes. The initial series of computations considered profiles in pressure and current profiles that are sustained by artificial sources. We use an equilibrium profile where the magnetic field starts with a winding number (safety factor) of 0.82 on axis. The resistive $1 / 1$ kink is linearly unstable and grows until it nonlinear generates other harmonics. The ensuing relaxation event modifies the profile near the magnetic axis, but the profiles tend to recover after the fluctuations are stabilized by the relaxation. This leads to the cyclical behavior shown in Figure 9. Details of the comparison with M3D are described in Ref. 5, where a more realistic computation is also defined for the next series of comparisons.

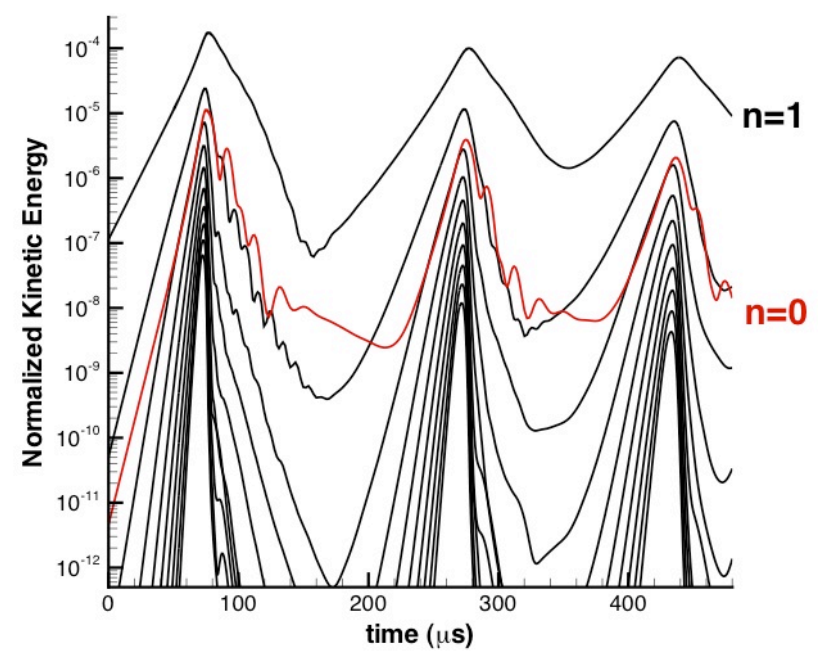

Figure 9. Evolution of kinetic fluctuation energy from the nonlinear NIMROD resistive MHD simulation for the benchmark series with the M3D code.

\section{References}

1. C. R. Sovinec, A. H. Glasser, T. A. Gianakon, D. C. Barnes, R. A. Nebel, S. E. Kruger, D. D. Schnack, S. J. Plimpton, A. Tarditi, M. Chu, and the NIMROD Team, J. Comput. Phys. 195, 355 (2004).

2. X. S. Li and J. W. Demmel, ACM Trans. Mathematical Software 29, 110 (2003).

3. See "SuperLU Solver Supercharges NIMROD," 2003 Annual Report of the National Energy Research Scientific Computing Center, p. 20.

[http://www.nersc.gov/news/annual_reports/annrep03/annrep03.pdf]

4. W. Park, E. V. Belova, G. Y. Fu, X. Z. Tang, H. R. Strauss, and L. E. Sugiyama, Phys. Plasmas 6, 1796 (1999).

5. J. A. Breslau, C. R. Sovinec, and S. C. Jardin, "An Improved Tokamak Sawtooth Benchmark for 3D Nonlinear MHD," 20th International Conference on Numerical Simulation of Plasmas, accepted for publication in Communications in Computational Physics (Global Science, Hong Kong).

6. C. R. Sovinec, T. A. Gianakon, E. D. Held, S. E. Kruger, D. D. Schnack, and the NIMROD Team, Phys. Plasmas 10, 1727 (2003).

7. D. S. Harned and Z. Mikic, J. Comput. Phys. 83, 1 (1989). 
8. C. R. Sovinec, D. D. Schnack, A. Y. Pankin, D. P. Brennan, H. Tian, D. C. Barnes, S. E. Kruger, E. D. Held, C. C. Kim, X. S. Li, D. K. Kaushik, S. C. Jardin, and the NIMROD Team, J. Phys.: Conf. Series 16, 25 (IoP, London, 2005).

9. C. C. Kim, C. R. Sovinec, S. E. Parker, and the NIMROD Team, Comp. Phys. Comm. 164, 448 (2004).

10. N. A. Murphy and C. R. Sovinec, Phys. Plasmas 15, 042313 (2008).

11 A. I. D. Macnab, R. D. Milroy, C. C. Kim, and C. R. Sovinec, Phys. Plasmas 14, 092503 (2007).

12 P. Zhu, D.D. Schnack, F. Ebrahimi, E.G. Zweibel, M. Suzuki, C.C. Hegna \& C.R. Sovinec, "The absence of complete FLR stabilization in extended MHD," submitted to Phys. Rev. Lett.

13. V. V. Mirnov, C. C. Hegna, and S. C. Prager, Phys. Plasmas 11, 4468 (2004).

\section{Products}

Journal Publications

1. C. R. Sovinec, T. A. Gianakon, E. D. Held, S. E. Kruger, D. D. Schnack, and the NIMROD Team, "NIMROD: a Computational Laboratory for Studying Nonlinear Fusion Magnetohydrodynamics," Physics of Plasmas 10, 1727 (2003).

2. C. R. Sovinec, A. H. Glasser, T. A. Gianakon, D. C. Barnes, R. A. Nebel, S. E. Kruger, D. D. Schnack, S. J. Plimpton, A. Tarditi, M. Chu, and the NIMROD Team, "Nonlinear Magnetohydrodynamics Simulation using High-Order Finite Elements," Journal of Computational Physics 195, 355 (2004).

3. E. D. Held, J. D. Callen, C. C. Hegna, C. R. Sovinec, T. A. Gianakon, and S. E. Kruger, "Nonlocal Closures for Plasma Fluid Simulations," Physics of Plasma 11, 2419 (2004).

4. S. E. Kruger, C. R. Sovinec, D. D. Schnack, and E. D. Held, "Free-Boundary Simulations of DIII-D Plasmas with the NIMROD Code," Computer Physics Communications 164, 34 (2004).

5. C. C. Kim, C. R. Sovinec, S. E. Parker, and the NIMROD Team, "Hybrid Kinetic-MHD Simulations in General Geometry," Computer Physics Communications 164, 448 (2004).

6. S. E. Kruger, D. D. Schnack, and C. R. Sovinec, "Dynamics of the Major Disruption of a DIII-D Plasma," Physics of Plasmas 12, 56113 (2005).

7. C. R. Sovinec, D. D. Schnack, A. Y. Pankin, D. P. Brennan, H. Tian, D. C. Barnes, S. E. Kruger, E. D. Held, C. C. Kim, X. S. Li, D. K. Kaushik, S. C. Jardin, and the NIMROD Team, "Nonlinear Extended Magnetohydrodynamics Simulation Using High-Order Finite Elements," Journal of Physics: Conference Series 16, 25 (IoP, London, 2005).

8. D. P. Brennan, S. E. Kruger, D. D. Schnack, C. R. Sovinec, and A. Pankin, "Computing Nonlinear Magnetohydrodynamic Edge Localized Instabilities in Fusion Plasmas," Journal of Physics: Conference Series 46, 63 (IoP, London, 2006).

9. D. D. Schnack, D. C. Barnes, C. C. Hegna, E. D. Held, C. C. Kim, S. E. Kruger, A. Y. Pankin, and C. R. Sovinec, "Computational Modeling of Fully Ionized Plasmas Using the Fluid Approximation,” Physics of Plasmas 13, 58103 (2006). 
10. P. Zhu, C. C. Hegna, and C. R. Sovinec, "Nonlinear Growth of a Line-Tied g-Mode near Marginal Stability," Physics of Plasmas 13, 102307 (2006).

11. P. Zhu, C. C. Hegna, C. R. Sovinec, A. Bhattacharjee, and K. Germaschewski, "Intermediate Nonlinear Regime of a Line-Tied g-mode," Physics of Plasmas 14, 055903 (2007).

12. C. R. Sovinec, D. C. Barnes, R. A. Bayliss, D. P. Brennan, E. D. Held, S. E. Kruger, A. Y. Pankin, D. D. Schnack, and the NIMROD Team, "Two-Fluid Studies of Edge Relaxation Events in Tokamaks," Journal of Physics: Conference Series 78, 012070 (IoP, London, 2007).

13. J. A. Breslau, C. R. Sovinec, and S. C. Jardin, "An Improved Tokamak Sawtooth Benchmark for 3D Nonlinear MHD," 20th International Conference on Numerical Simulation of Plasmas, accepted for publication in Communications in Computational Physics (Global Science, Hong Kong).

Conference Proceedings

1. V. V. Mirnov, F. Ebrahimi, R. Gatto, C. C. Hegna, S. C. Prager, C. R. Sovinec, P. W. Terry, and J. C. Wright, "Two-Fluid and Nonlinear Effects of Tearing and Pressure-Driven Resistive Modes in Reversed Field Pinches," in Proceedings of the Nineteenth International Conference on Plasma Physics and Controlled Nuclear Fusion Research, Lyon, France, 2002 (IAEA, Vienna, 2003).

2. C. R. Sovinec, A. Y. Pankin, S. E. Kruger, D. P. Brennan, P. B. Snyder, D. D. Schnack, E. D. Held, D. C. Barnes, G. Bateman, A. H. Kritz, S. C. Jardin, and J. Breslau, "Extended Magnetohydrodynamic Simulations of Edge Localized Modes in Existing and Future Tokamak Devices," in Proceedings of the 21st International Conference on Plasma Physics and Controlled Nuclear Fusion Research, Chengdu, China, 2006 (IAEA, Vienna, 2007).

3. V. V. Mirnov, C. C. Hegna, S. C. Prager, C. R. Sovinec, and H. Tian, "Two Fluid Dynamo and Edge-Resonant $\mathrm{m}=0$ Tearing Instability in Reversed Field Pinch," in Proceedings of the 21st International Conference on Plasma Physics and Controlled Nuclear Fusion Research, Chengdu, China, 2006 (IAEA, Vienna, 2007).

Invited Presentations

1. C. R. Sovinec, "NIMROD: a Computational Laboratory for Studying Nonlinear Fusion Magnetohydrodynamics," presented at the $44^{\text {th }}$ Annual Meeting of the Division of Plasma Physics of the American Physical Society, Orlando, Florida, 2002.

2. C. R. Sovinec, D. D. Schnack, A. Y. Pankin, D. P. Brennan, H. Tian, D. C. Barnes, S. E. Kruger, E. D. Held, C. C. Kim, X. S. Li, D. K. Kaushik, S. C. Jardin, and the NIMROD Team, "Nonlinear Extended Magnetohydrodynamics Simulation Using High-Order Finite Elements," Scientific Discovery through Advanced Computing, San Francisco, California, 2005.

3. C. R. Sovinec, D. C. Barnes, R. A. Bayliss, D. P. Brennan, E. D. Held, S. E. Kruger, A. Y. Pankin, D. D. Schnack, and the NIMROD Team, "Two-Fluid Studies of Edge Relaxation Events in Tokamaks," Scientific Discovery through Advanced Computing, Boston Massachusetts, 2007.

Web sites with additional information

- https://nimrodteam.org

- $\quad$ http://www.cptc.wisc.edu/sovinec research 


\section{- http://w3.pppl.gov/cemm}

Collaborations fostered

Prior to the SciDAC initiative, the multi-institutional NIMROD effort had been funded through a number of separate grants and contracts. The SciDAC support provided through CEMM established formal ties that helped ensure coordination of efforts by team members from Science Applications International Corporation, Tech-X Corporation, Utah State University, the University of Colorado at Boulder, and the University of Wisconsin-Madison. The organization of CEMM itself fostered collaborations between the NIMROD and M3D code development groups. The Wisconsin group has interacted with Drs. Stephen Jardin, Joshua Breslau, and Guoyong $\mathrm{Fu}$ of Princeton Plasma Physics Laboratory on algorithms for fluid-based plasma models and on benchmark applications. In addition, the SciDAC program encouraged cooperation between computer science groups and applications groups. Our interactions with TOPS researchers Dr. Dinesh Kaushik of Argonne National Laboratory and Dr. Xiaoye Li of Lawrence Berkeley National Laboratory led to very significant gains in computational performance for the NIMROD code.

\section{Computer modeling}

The NIMROD code solves fluid-based models of plasmas in linear and nonlinear initialvalue computations. To reproduce the extreme anisotropies that are characteristic of magnetized plasmas, NIMROD features high-order finite-element and Fourier basis functions, and this approach is effective even when the magnetic field becomes misaligned with the mesh. The twofluid algorithm staggers the flow velocity advance from the advances of number density, magnetic field and temperature to provide temporal centering without solving the entire system simultaneously. Our two-fluid model includes Braginskii gyroviscosity in the velocity advance, the Hall and electron-pressure-gradient terms in Ohm's law, and magnetization (cross) heat flows when electron and ion temperatures are advanced separately.

To avoid severe time-step limitations from the propagation of dispersive waves, like whistler and kinetic-Alfvén waves, each field advance is an implicit step. The 'implicit leapfrog' algorithm is analogous to semi-implicit MHD, but it requires solution of non-Hermitian algebraic systems. The strategy for preconditioning Krylov-space iteration, such as the conjugate gradient and generalized minimal residual methods, has been to use a separate direct solve over the poloidal plane for each Fourier component. This approach is effective for MHD simulations, but convergence is slow for the two-fluid magnetic-field advance in three-dimensional computations when variations over the toroidal angle develop. We are presently working on strategies to incorporate toroidal coupling in the preconditioning strategy. 